\title{
Cloning, Sequencing and Bioinformatic Analysis of Hypervariable Region of Hexon Gene of Avian Adenovirus 4 (AAV4) Associated with Angara Disease from Pakistan
}

\author{
Asma Jabeen $^{1,2^{*}}$, Khalid Naeem ${ }^{2}$, Naila Siddique ${ }^{3}$, Muhammad Athar Abass ${ }^{3}$, S.M. Saqlan Naqvi ${ }^{1}$ \\ ${ }^{1}$ Department of Biochemistry PMAS Arid Agriculture University Rawalpindi-46000, Pakistan \\ ${ }^{2}$ National Institute of Genomics and Advanced Biotechnology, National Agricultural Research Centre Islamabad-44000, \\ Pakistan \\ ${ }^{3}$ National Reference Laboratary for poultry diseases, Animal Sciences institute National Agricultural Research Centre \\ Islamabad-44000, Pakistan \\ *For Correspondence: asmajabeen2010@yahoo.com
}

\begin{abstract}
Angara Disease (AD), caused by avian adenovirus-4 (AAV-4), is an important viral disease of poultry causing heavy economic losses in the affected flocks. A field isolate of AAV-4 recovered from AD outbreak was isolated by growing in chicken embryo liver cells. The hypervariable region of hexon gene of AAV-4 was amplified by PCR. The amplified product was cloned in TA cloning vector and subjected to sequencing. Sequenced isolate showed 99\% homology with AAV-4 hexon gene sequences available in the NCBI GenBank and found to be clustered with Indian and Pakistan AAV-4 isolates. Sequenced hexon gene was analyzed using online bioinformatics tools. Probable primary secondary and 3D structures were predicted using on line ExPASy server. 26 B-cell epitopes were predicted using on line ABCpred server. Hypervariable region of hexon gene was predicted as a subunit vaccine candidate using vaxijen server with a score of 0.4865 showing it as probable antigen. (C) 2015 Friends Science Publishers
\end{abstract}

Keywords: Avian Adenovirus-4; Hydropericardium Syndrome; Phylogenetic analysis; Angara Disease

\section{Introduction}

Angara Disease also called hydropericardium syndrome (HPS) is an important disease of broilers (3-6 weeks old) and breeders (6-20 weeks old) which causes high mortality resulting in heavy economic losses. AD is characterized by the presence of transparent watery liquid in the pericardium, inflamed discolored fragile liver and congested kidneys (Ahmad et al., 1989). The disease was first time reported from Angara Goth Karachi, Pakistan, in 1987 giving the name as angara disease (Jaffery, 1988). AD is caused by Avian Adenovirus serotype-4 (AAV-4), a non-enveloped icosahederal DNA virus. The virus is composed of an outer protein shell and the core contains a double stranded DNA of $45 \mathrm{~kb}$ in size. The structural proteins of this adenovirus comprises of are hexons and pentons, out of which hexon is a major immunogenic protein. Hexon gene consists of conserved pedestal regions $\mathrm{P} 1$ and $\mathrm{P} 2$ and variable loop regions L1, L2 and L4. The exposed capsid is formed by variable loop region of hexon gene. Major nucleotide changes have been frequently observed in the variable loop region of hexon gene (Robert et al., 1986). Full length Hexon carries type, group and subgroup specific antigenic determinants, whereas, hypervariable region of hexon contains type specific antigenic regions. Therefore, it becomes important to understand the molecular and antigenic characteristics of hypervariable hexon of circulating AAV-4 in this country. For this purpose, Present study was designed to characterize the hypervariable region of the hexon gene from the field isolate of AAV-4 by cloning, sequencing, phylogenetic analysis, primary, secondary 3D structure analysis, epitope prediction and vaccine candidate characterization.

\section{Materials and Methods}

\section{Source of Virus}

A field isolate Pak/NARC-3317/2008 of AAV-4 recovered from HPS outbreak was used in this study. The virus was confirmed as HPS virus through the PCR and AGPT (Ganesh et al., 2002). 


\section{Virus Isolation in Cell Culture}

Avian adenovirus-4 was propagated in chicken embryo liver cell culture prepared from 16 days old chicken embryonated eggs using the protocol described by Naeem et al. (1995). AGPT, PCR and virus neutralization test were done for confirmation of propagated AAV-4.

\section{Primer Designing and PCR}

For cloning studies gene specific primers with restriction sites of EcoR1 and Not1were designed using hypervariable region of hexon (Ganesh et al., 2002). The viral DNA was extracted from cell culture propagated AAV-4 by means of Invitrogen DNA extraction kit. Hypervariable region of hexon was amplified. The PCR product was purified by Wizard SV Gel and PCR Clean up System kit (Promega) using manufacturer's instruction.

\section{Cloning and Sequencing}

Cloning of PCR product (Hypervariable region of Hexon gene) in $\mathrm{pTZ57R/T}$ was done by using InsTAclone PCR product cloning kit (Fermentas). Recombinant T/A vector was transformed into E.coli DH5 $\alpha$. Confirmation of recombinant plasmid was done by restriction digesion and sequencing. Recombinant plasmid was sent for sequencing to Macrogen (Korea).

\section{Phylogenetic Analysis}

The sequenced hexon gene was subjected to homology search using Basic Local Alignment Search Tool. Multiple sequence Alignment was done using clustalW and phylogenetic tree was constructed using MEGA 5.0. Neighbor joining method (NJ) was used to construct the tree. It calculated pairwise distance between sequences and formed groups of sequences that are similar (Harrison and Langdale, 2006). All sequences used for phylogenetic analysis were obtained from GenBank.

\section{Prediction of Proteins Physicochemical Properties and Structure}

Physicochemical properties of hexon protein were predicted by using online tool protparm. Primary, secondary and 3D structure of protein were predicted using expassy server (Gasteiger et al., 2005).

\section{Prediction of B-cell Epitopes}

Protein sequence was analyzed for the prediction of B-cell epitopes by ABCpred server using neural network (Saha et al., 2006). The vaccine candidate characterization of hexon was done using Vaxijen computational tool.

\section{Results}

\section{Isolation and Detection of AAV-4}

Avian adenovirus-4 was isolated in chicken embryo liver cell culture and cytopathic effects of AAV-4 were characterized by rounding and Clumping of infected cells. AGPT of cell culture propagated AAV-4 showed band with known hyperimmune serum. Virus neutralization and PCR test showed positive results.

\section{PCR Amplification and Cloning of the Hypervariable Region of Hexon Gene}

A single ampilcon of 730bp of hexon gene was observed on agarose gel (Fig. 1) eluted, purified and cloned. Recombinant colonies were screened for amplification of hexon gene by colony PCR (Fig. 2). Plasmid DNA was isolated from PCR positive colonies (Fig. 3). Restriction digesion of recombinant plasmid with E coRl and Notl confirmed the insertion of 730bp hexon gene (Fig. 4).

\section{Sequencing and Homology}

Nucleotide sequence of the cloned partial hexon of AAV-4 isolate revealed 730bp sequence Sequencing results revealed a complete open reading frame (ORF) of 727 bp. Partial hexon gene sequence was submitted to NCBI and assigned the accession numbers KM217572. BLAST (Basic Local Alignment Search Tool) revealed that nucleotide sequence of Pakistani AAV-4 isolate used in present study showed 99\% homology with Indian and Pakistani AAV-4 hexon gene available in NCBI Genbank (Haryana, Palampur, Izetnagar Indian Quail, NIAB1, Accession no FM210201.1, EU847626.1, AY581274.1 and FR686931.2). The HPS isolate showed more than $96 \%$ identity with FAV-10 (U26221.1).

\section{Phylogenetic Analysis}

Phylogenetic tree was constructed from the nucleotide sequences retrieved through BLAST. Phylogenetic analysis showed close relation between Indian and Pakistani FAdV-4 (Fig. 5). Pakistani AAV-4 isolate formed cluster with other AAV-4 Pakistani and Indian isolates (Haryana, Palampur, Izetnagar Indian Quail, NIAB1 Accession no FM210201.1, EU847626.1, AY581274.1 and FR686931.2).

\section{Prediction of Physicochemical Properties}

Physicochemical properties of hypervariable region of hexon protein were predicted by using online tool protparm (Table 1). Translation revealed a protein of 242 amino acids. Theoretical pI and, Aliphatic index of hexon protein from AAV-4 isolate are given in Table 1 . The instability index was computed to be 27.70 this classified the hexon protein as stable. The half-life of predicted hexon protein of AAV-4 
Table 1: Physicochemical properties of hypervariable region of hexon gene of AAV-4

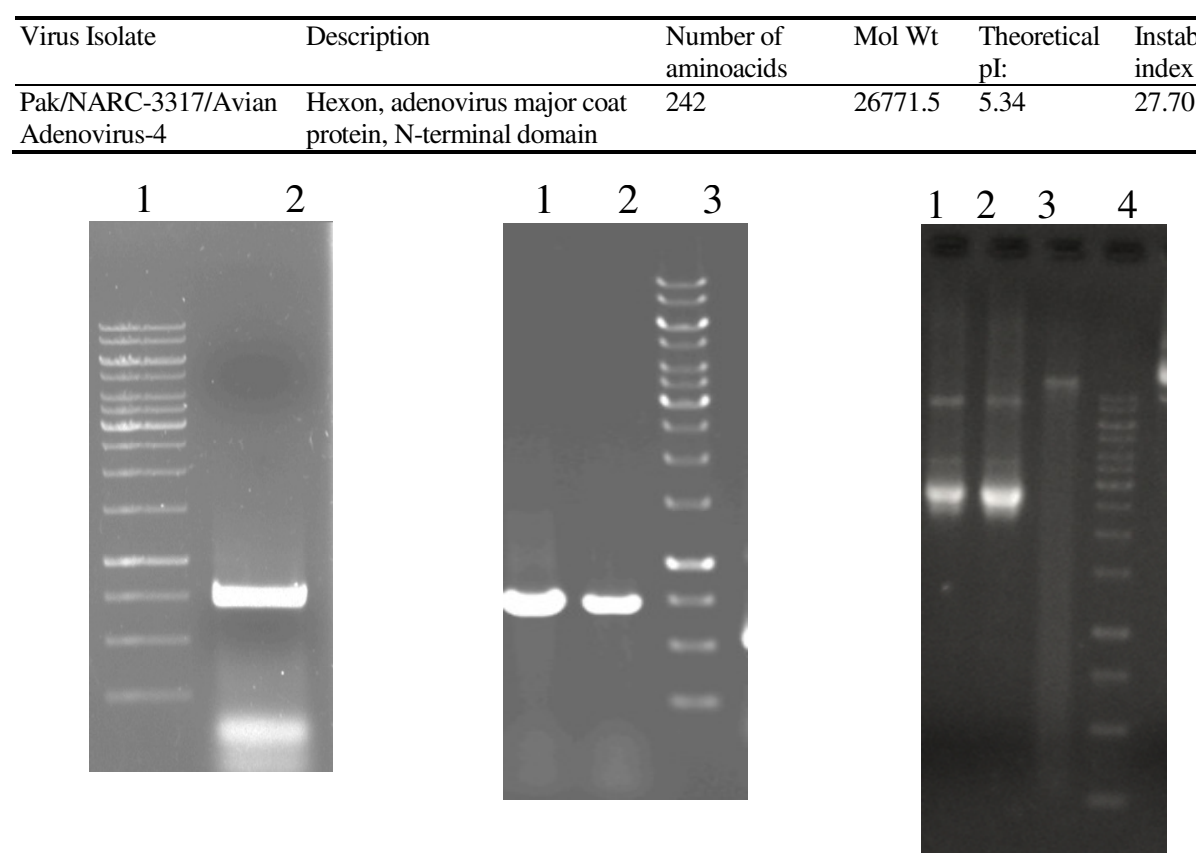

Fig. 1: Amplification of Fig. 2: Colony PCR Results hexon gene

Lane-1 Ladder 1kb; Lane-2 730bp Hexon gene AAV-4 Lane-1 730bp Hexon gene AAV-4 Clone-1; Lane-2 730bp Hexon gene AAV-4 clone-2; Lane-3 Ladder $1 \mathrm{~kb}$
Fig. 3: Plasmid Isolation Lane-1,2 Recombinant plasmid clone-1,2; Lane-3 -ve; Lane-4 Ladder $1 \mathrm{~kb}$

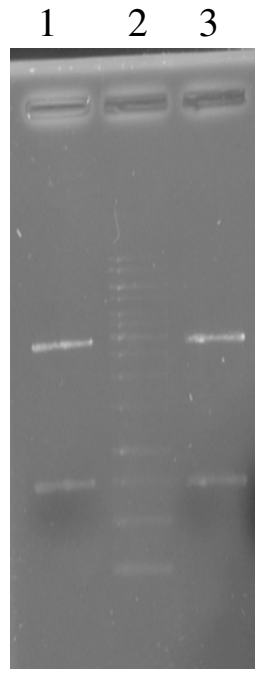

Fig. 4: Restriction digesion of recombinant plasmid Lane-1 Digested 730bp Hexon gene AAV-4 Clone-1; Lane-2 Ladder $1 \mathrm{~kb}$; Lane-3 Digested 730bp Hexon gene AAV-4 clone-2 isolate was estimated to be $>10 \mathrm{~h}$ (in E.coli, in vivo). Information on primary structure supported the possibility of expression of hexon gene in E. coli.

\section{Prediction of Protein Structure}

Partial hexon protein analysis was done using computational tools. The nucleotide sequence was imported to expasy server (www.expasy.org/tool) for primary structure analysis (Fig. 6). Insilico translation revealed a protein of 242 amino acids. Domain analysis was done by Interproscan which showed Adenovirus $\mathrm{N}$ terminal hexon domain (Fig. 7).

The sequence was further analyzed for secondary structure using online server PSIPRED. Predicted secondary structure showed high confidence of coiled structure and low confidence of helical structure (Fig. 8). Homology Modeling of hexon protein from field isolate of AAV-4 was done by using SWISSMODEL. The software used human adenovirus $2(\operatorname{HAd} 2)$ hexon protein structure as template to predict the 3D structure of hexon protein (Fig. 9).

B-cell epitopes of hypervariable region of hexon gene were predicted by using ABCpred server. All the epitopes were found to be on the surface and hydrophilic in nature, which is the characteristic of the B-cell epitopes. The protein was analyzed at a threshold setting of 0.5 . AAV-4 Isolate showed 26 epitopes (Table 2). Hypervariable region of hexon gene was predicted as a subunit vaccine candidate using Vaxijen server with a score of 0.4865 showing it as probable antigen.

\section{Discussion}

Hydropericardium syndrome is an important emerging disease with high mortality rate resulting in heavy economic losses in poultry. In the present study hypervariable region of the major immunogenic polypeptide (hexon) of the Pakistani field isolate of AAV-4 was amplified, cloned, sequenced and characterized by using various bioinformatics tools.

Hexon, is major structural protein and carries the type, group and sub-group specific antigenic determinants. Crawford-Miksza and Schnurr (1996) reported that the hypervariable regions of hexon gene carries type specific antigentic determinants and are 'hotspots' for recombination.

In this study L1 and P1 Loop of hexon gene was amplified by PCR and cloned in PTZ57R vector. Asthana $e t$ al. (2011) cloned hexon gene of an indian AAV-4 into pGEM-T Easy vector. Sequencing results of present study showed an ORF of approximately 242 amino acids. Phylogenetic analysis revealed clustering of Pakistani AAV4 isolate with other AAV-4 Pakistani isolates. Pakistani 
Table 2: Total Predicted Epitopes of partial hexon gene of AAV-4 by ABCpreD server

\begin{tabular}{lll}
\hline Sr No & Scoring range & No of Epitopes \\
\hline 1 & $0.51-0.60$ & 0 \\
2 & $0.61-0.70$ & 03 \\
3 & $0.71-0.80$ & 09 \\
4 & $0.81-0.90$ & 07 \\
5 & $0.91-0.99$ & 07 \\
\hline
\end{tabular}

$$
\text { Fowl adenovirus serotype } 4 \text { from Pakistan }
$$

Fig. 5: Phylogenetic tree of hypervariable region of hexon gene

MGST YFDIKGILDRGPSFK PYCGTA YNPLAPKES MFNNWSETA PGQNVSASGQLSNVYTNTSTSKDTTAAQVTKISGVFPNPNQGP GRNPLRRVQNANTGVLGRFAKSQYNYAYGAYVKPVAADGSQ SLTQTPYWIMDNTGTNYLGAVAVEDYTNSLSYPDTIVVPPPED YDDYNIGTTRALR PNYIGFRDNFINLLYHDSGVCSGTLNSERS GMNVVVELPDR TELS Y QYMLADMMSRHH

Fig. 6: Predicted Primary Structure of Hypervariable region of hexon gene of $\mathrm{AAV}-4$

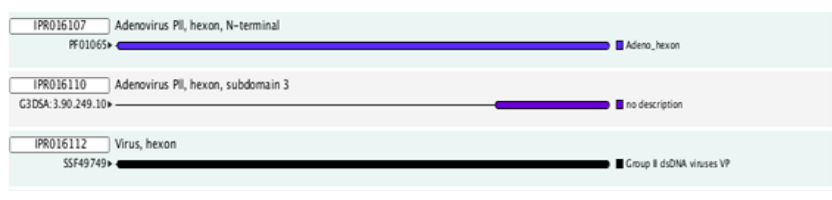

Fig. 7: Domain Analysis of Hypervariable region of hexon gene of AAV-4

HPS isolate showed 99\% homology to Indian HPS isolates. Our results are in line with findings of Shah et al. (2011) who found that the nucleotide sequence Pakistani isolate has 99\% identity with several Indian isolates of FAdV-4. Phylogenetic analysis showed close relation between Indian and Pakistani FAdV-4. So it was found that isolates of Avian adenovirus-4 in India and Pakistan had a common ancestor.

Physicochemical properties using bioinformatic tools classified the hexon protein as stable. The half-life of predicted hexon protein showed possibility of expression of hexon gene in E.coli. Primary structure revealed a protein of 242 amino acids. Predicted secondary structure showed high confidence of coiled structure. Predicted 3D structure of hypervariable region of hexon gene by procheck programs showed good energy profile and stereochemistry with no residues in the disallowed regions on Ramachandran plot. AAV-4 Isolate showed 26 epitopes, Hypervariable region of hexon gene was predicted as a subunit vaccine candidate

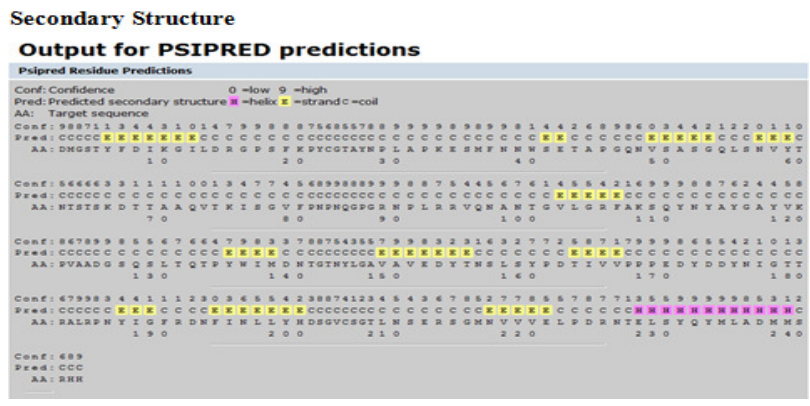

Fig. 8: Predicted seconday Structure of Hypervariable region of hexon gene of AAV-4

\section{D Structure}

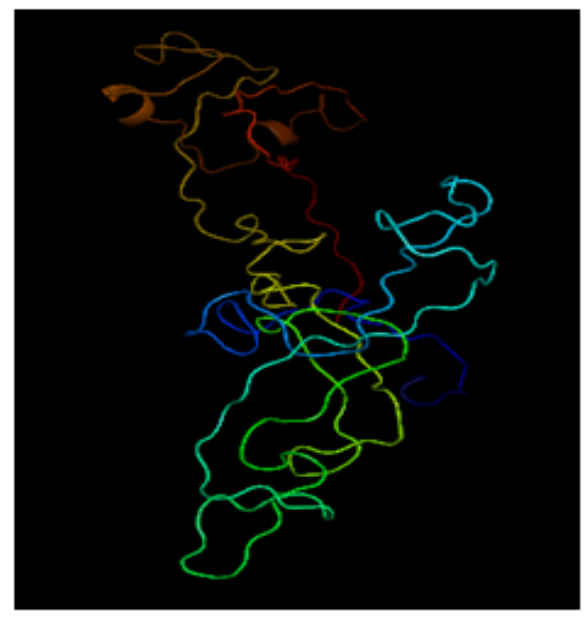

Fig. 9: Predicted 3D Structure of Hypervariable region of hexon gene of AAV-4

using Vaxijen server showing it as probable antigen.

In conclusion present study successfully characterized hypervariable region of hexon gene from Pakistani field isolate of AAV-4 by cloning, sequencing, Phylogenetic and bioinformatic analysis. Epitope prediction and vaccine candidate characterization have been reported first time for Pakistani field isolate of AAV-4 and could eventually be proposed as an important component of immunodiagnostics and subunit vaccine for future studies.

\section{Acknowledgements}

The Author acknowledges financial grant from National Reference Labortary for poultry Diseases, Islamabad, Pakistan.

\section{References}

Ahmad, I., M. Afzal, M.I. Malik, Z. Hussain and W. Hanif, 1989. Studies on the disease pattern and etiology of hydropericardium syndrome (Angara disease) in broiler chickens in Pakistan. Pak. J. Agric. Res., 10: $195-199$ 
Asthana, M., V.K. Singh, R. Kumar and R. Chandra, 2011. Isolation, Cloning and In silico Study of Hexon Gene of Fowl Adenovirus 4 (FAV4) Isolates Associated with Hydro Pericardium Syndrome in Domestic Fowl. J. Proteomics Bioinform., 4: 190-195

Crawford-Miksza, L. and D.P. Schnurr, 1996. Analysis of adenovirus hexon proteins reveals the location and structure of seven hypervariable regions containing serotype-specific residues. J. Virol., 70: 1836-1844

Ganesh, K., V.V. Suryanarayana and R. Raghavan, 2002. Detection of fowl adenovirus associated with hydropericardium hepatitis syndrome by a polymerase chain reaction. Vet. Res. Commun., 26: 73-80

Gasteiger, E., C. Hoogland, A. Gattiker, S. Duraud and M.R. Wilkins, 2005. In: Protein Identification and Analysis Tools on the ExPAsy Server, pp: 571-607. John, M. and M. Walker (eds.). The Proteomics Protocols Handbook, Humana Press, New York, USA

Harrison, C.J. and J.A. Langdale, 2006. A step by step guide to phylogeny reconstruction. Plant J., 45: 561-572
Jaffery, M.S., 1988. Atreatiseon Angara disease (hydropericardium-pulmonary oedema hepatonephritis syndrome). J. Pak. Vet. Med. Ass., 34: 1-33

Naeem, K., T. Niazi, S.A. Malik and A.H. Cheema, 1995. Immunosuppressive potential and pathogenecity of an avian adenovirus isolate involved in hydropericardium syndrome in broilers. Avian Dis., 39: 723-728

Robert, M.M., J.L. White, M.G. Grutter and R.M. Burnett, 1986. Threedimensional structure of the adenovirus major coat protein hexon. Science, 232: 1148-1151

Saha, S. and G.P. Raghava, 2006. Prediction of continuous B-cell epitopes in an antigen using recurrent neural network. Proteins, 65: 40-48

Shah, M.S., A. Ashraf, M. Khan, M. Rahman, M. Habib, S.1. Babapoor, A. Ghaffar, I.R. Malik, S.A. Khannum and J.A. Qureshi, 2011. Molecular characterization of fowl adenoviruses associated with hydro-pericardium syndrome in broilers. Afr. J. Micrbiol. Res., 30: 5407-5414

(Received 28 August 2014; Accepted 24 November 2014) 